Wright State University

CORE Scholar

Mathematics and Statistics Faculty

Publications

Mathematics and Statistics

$1-1992$

\title{
Boundary Velocity Control of Incompressible-Flow with an Application to Viscous Drag Reduction
}

\author{
Max D. Gunzberger \\ Lisheng Hou \\ Tom Svobodny \\ Wright State University - Main Campus, thomas.svobodny@wright.edu
}

Follow this and additional works at: https://corescholar.libraries.wright.edu/math

Part of the Applied Mathematics Commons, Applied Statistics Commons, and the Mathematics Commons

\section{Repository Citation}

Gunzberger, M. D., Hou, L., \& Svobodny, T. (1992). Boundary Velocity Control of Incompressible-Flow with an Application to Viscous Drag Reduction. SIAM Journal on Control and Optimization, 30 (1), 167-181. https://corescholar.libraries.wright.edu/math/33

This Article is brought to you for free and open access by the Mathematics and Statistics department at CORE Scholar. It has been accepted for inclusion in Mathematics and Statistics Faculty Publications by an authorized administrator of CORE Scholar. For more information, please contact library-corescholar@wright.edu. 


\title{
BOUNDARY VELOCITY CONTROL OF INCOMPRESSIBLE FLOW WITH AN APPLICATION TO VISCOUS DRAG REDUCTION*
}

\author{
MAX D. GUNZBURGER †, LISHENG HOU $\ddagger$, AND THOMAS P. SVOBODNY§
}

\begin{abstract}
An optimal boundary control problem for the Navier-Stokes equations is presented. The control is the velocity on the boundary, which is constrained to lie in a closed, convex subset of $H^{1 / 2}$ of the boundary. A necessary condition for optimality is derived. Computations are done when the control set is actually finite-dimensional, resulting in an application to viscous drag reduction.
\end{abstract}

Key words. optimal control, Navier-Stokes equations, boundary control, finite element methods, distributed parameter systems

AMS(MOS) subject classifications. 49A22, 49B22, 49D05, 65N30, 76D05, 93C10, 93C20

1. Introduction. We are concerned with a constrained optimization problem for steady fluid flow, namely that of computing a boundary value of the velocity that minimizes a volume integral that represents frictional energy dissipation. The constraint is the system of equations for viscous incompressible flow. The boundary value of the velocity, hereupon dubbed the control, is constrained to a closed, convex subset of $\mathbf{H}^{1 / 2}\left(\Gamma_{c}\right)$, where $\Gamma_{c}$ is a portion of the body boundary. Such a constraint is necessary since the control does not appear explicitly in the cost functional. Moreover, we cannot eliminate the control in solving coupled state-adjoint equations. In the present case a minimum principle gives us a variational inequality that couples the system of state and adjoint equations. If we further constrain the control set to be in a finite-dimensional subspace, then we are led to an optimization problem where the feasibility set is the set of vertices of a cube in $m$-dimensional Euclidean space.

Our choice of the cost functional to be optimized is motivated by the following physical consideration (cf. [9]). If a body with boundary $\Gamma$ is immersed in a fluid, then the force acting on the body is

$$
\mathbf{F}=\int_{\Gamma} \mathbf{T} \cdot \mathbf{n} d \Gamma
$$

where $\mathbf{T}$ is the stress tensor and $\mathbf{n}$ is the unit normal vector pointing into the body. If we consider a body moving with rectilinear velocity $\mathbf{V}$, then a measure of the component of the force in the direction of the velocity is

$$
\mathbf{F}_{D}=\mathbf{F} \cdot \mathbf{V}=\mathbf{V} \cdot \int_{\Gamma} \mathbf{T} \cdot \mathbf{n} d \Gamma=\int_{\Gamma} \mathbf{v} \cdot \mathbf{T} \cdot \mathbf{n} d \Gamma
$$
26, 1990.

*Received by the editors March 13, 1989; accepted for publication (in revised form) October

†Department of Mathematics, Virginia Polytechnic Institute and State University, Blacksburg, Virginia 24061. The work of this author was supported by Air Force Office of Scientific Research grants AFOSR-88-0197 and AFOSR-90-0179.

‡Départment of Mathématiques et de Statistique, Université Laval, Québec, G1K 7P4. The work of this author was supported by the Department of Education of the Province of Québec, Actions Structurantes Program.

$\S$ Department of Mathematics and Statistics, Wright State University, Dayton, Ohio 45435. The work of this author was supported by Air Force Office of Scientific Research grants AFOSR-86-0085 and AFOSR-85-0263 while he was visiting Virginia Tech. 
where $\mathbf{v}=\mathbf{v}(\mathbf{x}, t)$ is the velocity field of the fluid. If we assume the linear constitutive law, i.e., an expression for stress is given by

$$
\mathbf{T}=-p \mathbf{I}+2 \mu \mathbf{D}
$$

where $p$ denotes a pressure field and $\mu$ the (constant) viscosity of the fluid, and $\mathbf{D}=\mathbf{D}(\mathbf{v})=\frac{1}{2}\left(\nabla \mathbf{v}+\nabla \mathbf{v}^{T}\right)$ is a symmetric tensor of first-order derivatives of $\mathbf{v}$, an integration by parts gives

$$
\mathbf{F}_{D}=2 \mu \int_{\Omega} \mathbf{D}: \mathbf{D} d x-\int_{\Omega} p(\operatorname{div} \mathbf{v}) d x+\mu \int_{\Omega} \mathbf{v} \cdot \nabla(\operatorname{div} \mathbf{v}) d x
$$

(We are assuming that the fluid is isotropic, reflected in the fact that the medium itself is described by the scalar quantity $\mu$.) Although this expression for the drag was derived in the context of the ideal conditions mentioned, it seems reasonable to use the right-hand side as a cost functional to be minimized subject to the constraints imposed by the incompressible Navier-Stokes equations. Of course, on this constraint set, the second and third terms on the right-hand side are clearly zero, thus making our cost functional positive. The term to which this functional then reduces is known in the literature as the dissipation function; it represents the rate at which heat energy is conducted into the fluid, or equivalently, the rate at which heat is generated by deformations of the velocity field.

We apply our results to the following problem. It is known that for an aerodynamic body moving at uniform velocity, the main contribution to retardation is the frictional force. This force is increased if the boundary layer becomes turbulent. In addition, the body may have to overcome adverse pressure gradients if the boundary layer separates. We want to reduce viscous (skin-friction) drag of an immersed body whose relative velocity with respect to the fluid is fixed $\left(\mathbf{v}_{\infty}\right)$. In the specific application that is considered at the end, the method of control is the following: we have $m$ disjoint regions (holes) on the surface of the body where we can specify positive (blowing) or negative (sucking) velocities [4]. Of course, the rate of flow at each hole is strictly limited, not only for the obvious reasons, but because too strong a control would change the lift, and the problem would not represent the one posed. On the other hand, the control velocity must dominate pressure gradients. We will assume that the control set is small enough so that the velocity profile at each hole is fixed, and thus the problem reduces to a finite-dimensional control problem.

The numerical approximation is carried out using the finite-element method; a sketch of the procedure as well as a model problem is given at the end. Further details concerning the approximation of such optimization problems as well as the more difficult (from the viewpoint of approximation) unconstrained case, including error estimates, are given in [3]. The authors are presently applying the techniques and algorithms discussed here to other applications, e.g., flows about airfoils.

1.1. Notation. Real $k$-dimensional Euclidean space is denoted $R^{k} ; R_{+}^{k}$ is the subset with nonnegative coordinates. The domain $\Omega$ is a bounded smooth domain in $R^{2}$ or $R^{3}$, whose boundary consists of two connected components $\Gamma=\Gamma_{e} \cup \Gamma_{c}$. Furthermore, $\Gamma_{e}=\Gamma_{(1)} \cup \Gamma_{(2)}$, where $\Gamma_{(1)}$ must have an interior, but $\Gamma_{(2)}$ may be empty. Let $\mathbf{H}^{m}(B)$ be the Hilbert space of $R^{n}$-valued functions defined on $B$ whose $j$ th derivatives, $0 \leq j \leq m$ are in $L^{2}(B)$. The norm in this space will be denoted by $\|\cdot\|_{m}$. In all cases, boldface indicates vector-valued. We will use the quotient spaces $L_{0}^{2}(\Omega)=\left\{f \in L^{2}(\Omega): \int_{\Omega} f d x=0\right\}$ and $\mathbf{H}_{0}^{1 / 2}(G)=\left\{\mathbf{g} \in \mathbf{H}^{1 / 2}(G): \int_{G} \mathbf{g} \cdot \mathbf{n} d G=0\right\}$. 
$<\cdot, \cdot>_{X}$ will mean the duality pairing on $X^{\prime} \times X$, while $\left.<<\cdot, \cdot\right\rangle_{X}$ will mean the inner product in the Hilbert space $X$.

The matrix $\left\{\partial u_{i} / \partial x_{j}\right\}$ will be denoted $\nabla \mathbf{u}$. The velocity deformation tensor is $\mathrm{D}(\mathbf{u})=\frac{1}{2}\left(\nabla \mathbf{u}+\nabla \mathbf{u}^{T}\right)$. We use the forms

$$
\begin{aligned}
a(\mathbf{u}, \mathbf{v}) & =2 \nu \int_{\Omega} \mathbf{D}(\mathbf{u}): \mathbf{D}(\mathbf{v}) d x \quad \forall \mathbf{u}, \mathbf{v} \operatorname{inH}^{1}(\Omega), \\
b(\mathbf{u}, p) & =-\int_{\Omega} p(\operatorname{div} \mathbf{u}) d x \quad \forall p \in L_{0}^{2}(\Omega), \quad \mathbf{u} \in \mathbf{H}^{1}(\Omega), \\
c(\mathbf{u}, \mathbf{v}, \mathbf{w}) & =\int_{\Omega} \mathbf{u} \cdot \nabla \mathbf{v} \cdot \mathbf{w} d x \quad \forall \mathbf{u}, \mathbf{v}, \mathbf{w} \in \mathbf{H}^{1}(\Omega) .
\end{aligned}
$$

The following estimate, which is a straightforward application of the Hölder and Sobolev inequalities (cf. [11]), will prove to be useful:

$$
|c(\mathbf{u}, \mathbf{v}, \mathbf{w})| \leq\left\{\begin{array}{l}
\text { const. }\|\mathbf{u}\|_{1 / 2}\|\mathbf{v}\|_{1}\|\mathbf{w}\|_{1} \\
\text { const. }\|\mathbf{u}\|_{1}\|\mathbf{v}\|_{1}\|\mathbf{w}\|_{1 / 2}
\end{array} .\right.
$$

These forms induce the following operators.

$$
A: \mathbf{H}^{1}(\Omega) \rightarrow \mathbf{H}^{-1}(\Omega)
$$

defined by

$$
\begin{gathered}
<A \mathbf{u}, \mathbf{v}>=a(\mathbf{u}, \mathbf{v}), \quad \mathbf{u} \in \mathbf{H}^{1}(\Omega), \quad \mathbf{v} \in \mathbf{H}_{0}^{1}(\Omega), \\
B: \mathbf{H}^{1}(\Omega) \rightarrow L_{0}^{2}(\Omega)
\end{gathered}
$$

defined by

$$
\begin{gathered}
<B \mathbf{u}, p>=b(\mathbf{u}, p), \quad \mathbf{u} \in \mathbf{H}^{1}(\Omega), \quad p \in L_{0}^{2}(\Omega), \\
\bar{B}: L_{0}^{2}(\Omega) \rightarrow \mathbf{H}^{-1}(\Omega)
\end{gathered}
$$

defined by

$$
<\bar{B} p, \mathbf{u}>=b(\mathbf{u}, p) \quad \mathbf{u} \in H_{0}^{1}(\Omega), \quad p \in L_{0}^{2}(\Omega)
$$

and

$$
C: \mathbf{H}^{1}(\Omega) \times \mathbf{H}^{1}(\Omega) \rightarrow \mathbf{H}^{-1}(\Omega)
$$

defined by

$$
<C(\mathbf{u}, \mathbf{v}), \mathbf{w}>=c(\mathbf{u}, \mathbf{v}, \mathbf{w}), \quad \mathbf{u}, \mathbf{v} \in \mathbf{H}^{1}(\Omega), \quad \mathbf{w} \in \mathbf{H}_{0}^{1}(\Omega) .
$$

For theorems concerning operators associated with the stationary Navier-Stokes equations, consult [7]. We will use the trace operators

$$
\gamma_{e}: \mathbf{H}^{1}(\Omega) \rightarrow \mathbf{H}^{1 / 2}\left(\Gamma_{e}\right), \quad \gamma_{c}: \mathbf{H}^{1}(\Omega) \rightarrow \mathbf{H}^{1 / 2}\left(\Gamma_{c}\right)
$$

and $\gamma=\gamma_{e} \times \gamma_{c}$. Optimal solutions of the optimization problem will be tagged with asterisks, $\mathbf{v}^{*}$, or with hats, $\hat{\mathbf{v}}$.

2. The optimization problem: boundary velocity control with constraints. We recall that we are faced with the problem of minimizing the functional $\left(\mathbf{F}_{D}\right)$, subject to the constraint

$$
-\nu \Delta \mathbf{v}+(\mathbf{v} \cdot \nabla) \mathbf{v}+\nabla p=0
$$




$$
\nabla \cdot \mathbf{v}=0
$$

and either of the sets of boundary conditions

$$
\left.\mathbf{v}\right|_{\Gamma_{e}}=\mathbf{v}_{\infty}, \quad \text { and }\left.\quad \mathbf{v}\right|_{\Gamma_{c}}=\mathbf{g}
$$

(the Boundary Value Control Problem 1), or

$$
\left.\mathbf{v}\right|_{\left(\Gamma_{1}\right)}=\mathbf{v}_{\infty},\left.\quad \mathbf{T}(\mathbf{v}) \cdot \mathbf{n}\right|_{\left(\Gamma_{2}\right)}=0, \quad \text { and }\left.\quad \mathbf{v}\right|_{\Gamma_{c}}=\mathbf{g}
$$

(the Boundary Value Control Problem 2), where the control function $\mathbf{g}$ is to lie in $\mathcal{U}$, a closed,convex subset of $\mathbf{H}^{1 / 2}\left(\Gamma_{c}\right)$, and where $\nu$ is the kinematic viscosity.

Our program is a straightforward approach to a constrained optimization problem: we investigate existence of optimal solutions, we verify the existence of Lagrange multipliers, and then we derive a minimum principle that couples the Euler-Lagrange equations as necessary conditions for the optimal boundary velocity. The cost functional is given by

$$
J(\mathbf{v})=\frac{\nu}{2} \int_{\Omega}\left|\nabla \mathbf{v}+\nabla \mathbf{v}^{T}\right|^{2} d x
$$

2.1. Existence of an optimal solution. Consider the Navier-Stokes equations in the weak form,

$$
\begin{gathered}
a(\mathbf{v}, \mathbf{w})+c(\mathbf{v}, \mathbf{v}, \mathbf{w})+b(\mathbf{w}, p)=0 \quad \forall \mathbf{w} \in \mathbf{H}_{0}^{1}(\Omega) \\
b(\mathbf{v}, q)=0 \quad \forall q \in L_{0}^{2}(\Omega) \\
\left.\mathbf{v}\right|_{\Gamma_{c}}=\mathbf{g}
\end{gathered}
$$

and either

$$
\left.\mathbf{v}\right|_{\Gamma_{e}}=\mathbf{v}_{\infty}
$$

or

$$
\left.\mathbf{v}\right|_{\Gamma_{(1)}}=\left.\mathbf{v}_{\infty} \quad \mathbf{T}(\mathbf{v}) \cdot \mathbf{n}\right|_{\Gamma_{(2)}}=0
$$

Always, $\mathbf{v}_{\infty} \in \mathbf{H}^{1 / 2}\left(\Gamma_{e}\right)$ (or $\mathbf{v}_{\infty} \in \mathbf{H}^{1 / 2}\left(\Gamma_{(1)}\right)$ in the case of (BVC2)) and, in the case of (BVC1), we assume

$$
\int_{\Gamma_{c}} \mathbf{g} \cdot \mathbf{n} d \Gamma+\int_{\Gamma_{e}} \mathbf{v}_{\infty} \cdot \mathbf{n} d \Gamma=0 .
$$

We consider the case (BVC1), and for simplicity assume that

$$
\int_{\Gamma_{e}} \mathbf{v}_{\infty} \cdot \mathbf{n} d \Gamma=0
$$


so that

$$
\int_{\Gamma_{c}} \mathbf{g} \cdot \mathbf{n} d \Gamma=0
$$

(Cases (BVC2) and (BVC1) without the above assumption can be treated in similar manner.)

DEFINITION 1. We define the admissibility set

$$
\begin{aligned}
\mathcal{T}_{\text {ad }}= & \left\{(\mathbf{v}, \mathbf{g}) \in \mathbf{H}^{1}(\Omega) \times \mathcal{U}\left(\subseteq \mathbf{H}^{1 / 2}\left(\Gamma_{c}\right)\right): J(\mathbf{v})<\infty \text { and there exists } p \in L_{0}^{2}(\Omega)\right. \\
& \text { and so that }(2.2)-(2.4) \text { and either }(2.4) \text { or }(2.5) \text { are satisfied }\} .
\end{aligned}
$$

Definition 2. We define an optimal solution $\left(\mathbf{v}^{*}, \mathbf{g}^{*}\right)$ to be one for which $J\left(\mathbf{v}^{*}\right) \leq$ $J(\mathbf{v})$ for any $(\mathbf{v}, \mathbf{g}) \in \mathcal{T}_{\text {ad }}$.

Definition 3. We define a local minimum $(\hat{\mathbf{v}}, \hat{\mathbf{g}})$ to be one for which there exists $\epsilon>0$ such that $J(\hat{\mathbf{v}}) \leq J(\mathbf{v})$ for any $(\mathbf{v}, \mathbf{g}) \in \mathcal{T}_{\text {ad }}$ with $\|\mathbf{v}-\hat{\mathbf{v}}\|_{1}<\epsilon$

LEMMA 2.1. There exists an optimal solution to the above problem.

Proof. First, note that $\mathcal{T}_{\text {ad }}$ is nonempty. This follows from the fact

$$
J(\mathbf{v}) \leq \nu\|\mathbf{v}\|_{1}^{2}
$$

and well-known existence theorems for solutions of stationary Navier-Stokes equations (cf. [1], [8], and [10]). Now let $\mathbf{V}_{m}=\left(\mathbf{v}_{m}, \mathbf{g}_{m}\right)$ be a sequence in $\mathcal{T}_{\text {ad }}$ such that

$$
\lim _{m \rightarrow \infty} J\left(\mathbf{v}_{m}\right)=\inf _{v \in \mathcal{T}_{\text {ad }}} J(\mathbf{v})
$$

By definition of $\mathcal{T}_{\text {ad }}, J\left(\mathbf{v}_{m}\right) \leq C_{0}$, a constant independent of $m$. On the boundary, $\mathbf{v}=\mathbf{v}_{\infty} \in \mathbf{H}^{1 / 2}\left(\Gamma_{e}\right)$, and because of the assumption on $\Gamma_{e}$ we have

$$
J(\mathbf{v}) \geq c_{1}\|\mathbf{v}\|_{1}^{2}-c_{2}\|\mathbf{v}\|_{1}-c_{3}
$$

(this is a direct consequence of Korn's inequality, cf. [2, p.117]); thus, $\left\|\mathbf{v}_{m}\right\|_{1} \leq C_{V}$. By the trace theorem, $\left\|\mathbf{g}_{m}\right\|_{1 / 2} \leq C_{U}$. We therefore have the weak limits

$$
\mathbf{v}_{m} \rightarrow \mathbf{v}^{*} \in \mathbf{H}^{1}(\Omega), \quad \mathbf{g}_{m} \rightarrow \mathbf{g}^{*} \in \mathbf{H}^{1 / 2}\left(\Gamma_{C}\right) .
$$

Since $\mathbf{H}^{1}(\Omega)$ imbeds compactly in $\mathbf{L}^{2}(\Omega)$, we also have the strong limit

$$
\mathbf{v}_{m} \rightarrow \mathbf{v}^{*} \in \mathbf{L}^{2}(\Omega)
$$

As $\mathcal{U}$ is convex, closed, it is closed in the weak topology, thus $\mathrm{g}^{*} \in \mathcal{U}$. Next, using the fact that $J$ is bounded, convex, we have that it is weakly lower semicontinuous, and thus $J\left(\mathbf{v}^{*}\right)=\inf _{v \in \mathcal{T}_{\text {ad }}} J(\mathbf{v})$.

Now we must show that the limit $\mathbf{v}^{*}$ is in the admissible set, $\mathcal{T}_{\text {ad }}$. We first show that $\mathbf{v}^{*}$ satisfies the boundary conditions. Since

$$
\gamma_{e} v_{m}=\mathbf{v}_{\infty}, \quad \gamma_{c} \mathbf{v}_{m}=\mathbf{g}_{m}
$$

we want to show that $\gamma_{e} \times \gamma_{c}$ is a weakly closed operator. But it is continuous on $\mathbf{H}^{1}(\Omega)$ and so it is closed, and thus has a closed graph, and thus by the Hahn-Banach theorem, is weakly closed. Thus

$$
\gamma_{e} \mathbf{v}^{*}=\mathbf{v}_{\infty}, \quad \gamma_{c} \mathbf{v}^{*}=\mathbf{g}^{*}
$$


Now we show that the limiting flow is incompressible. The condition

$$
b\left(\mathbf{v}_{m}, q\right)=0, \quad \forall q \in \mathbf{L}^{2}
$$

means that $\mathbf{v}_{m} \in \operatorname{ker}(B)$ forall $m$. But since $\operatorname{ker}(B)$ is a closed subspace, it is weakly closed, and so

$$
\mathbf{v}^{*} \in \operatorname{ker}(B) \Rightarrow b\left(\mathbf{v}^{*}, q\right)=0 \quad \forall q \in L^{2}(\Omega) .
$$

It is clear that $a\left(\mathbf{v}_{m}, \mathbf{w}\right) \rightarrow a\left(\mathbf{v}^{*}, \mathbf{w}\right)$ forall $w \in \mathbf{H}^{1}(\Omega)$. On the other hand, the operator $C(\cdot, \cdot)$ is weak sequentially continuous. To see this, take $\mathbf{w} \in \mathbf{C}_{0}^{\infty}(\Omega)$, and consider

$$
c\left(\mathbf{v}_{m}, \mathbf{v}_{m}, \mathbf{w}\right)=-c\left(\mathbf{v}_{m}, \mathbf{w}, \mathbf{v}_{m}\right)=-\sum_{i, j} \int_{\Omega} v_{m i} \frac{\partial w_{j}}{\partial x_{i}} v_{m j} d x .
$$

Since $(\nabla \mathbf{w})_{i j} \in L^{\infty}(\Omega)$, and since $\mathbf{v}_{m} \rightarrow \mathbf{v}^{*}$ in $\mathbf{L}^{2}(\Omega)$, we have

$$
c\left(\mathbf{v}_{m}, \mathbf{v}_{m}, \mathbf{w}\right) \rightarrow c\left(\mathbf{v}^{*}, \mathbf{v}^{*}, \mathbf{w}\right) \quad \forall \mathbf{w} \in \mathbf{C}_{0}^{\infty}
$$

but this space is dense in $\mathbf{H}_{0}^{1}(\Omega)$, thus

$$
A \mathbf{v}_{m}+C\left(\mathbf{v}_{m}, \mathbf{v}_{m}\right) \rightarrow A \mathbf{v}^{*}+C\left(\mathbf{v}^{*}, \mathbf{v}^{*}\right) \in \mathbf{H}^{-1}
$$

Morevoer, $A \mathbf{v}_{m}+C\left(\mathbf{v}_{m}, \mathbf{v}_{m}\right) \in R(\bar{B})$ for all $m$; but the inf sup condition ([7, p.81]) implies that $R(\bar{B})$ is closed, and thus

$$
A \mathbf{v}^{*}+C\left(\mathbf{v}^{*}, \mathbf{v}^{*}\right) \in R(\bar{B})
$$

or, in other words, there exists a $p^{*} \in L_{0}^{2}(\Omega)$ such that

$$
A \mathbf{v}^{*}+C\left(\mathbf{v}^{*}, \mathbf{v}^{*}\right)=-\bar{B} p^{*}
$$

which completes the proof of existence of an optimal control.

2.2. The Lagrange multiplier rule. The idea of the technique of Lagrange multipliers is that the problem of minimizing a functional subject to a constraint can be reduced to the unconstrained minimization of an auxiliary functional, the Lagrangian. Important in applications of convex optimization is the existence of a minimum principle. (The general theory of the parametrized Lagrange multiplier rule is developed in [5, Chap. 1] and [13, Chap. 1].)

We can define a mapping

$$
\mathbf{F}: \mathbf{H}^{1}(\Omega) \times L_{0}^{2}(\Omega) \times \mathcal{U}_{\mathrm{ad}} \rightarrow \mathbf{H}^{-1}(\Omega) \times L_{0}^{2}(\Omega) \times \mathbf{H}^{1 / 2}\left(\Gamma_{e}\right) \times \mathbf{H}^{1 / 2}\left(\Gamma_{c}\right)
$$

by

$$
\begin{aligned}
& F^{1}(\mathbf{v}, p, \mathbf{g})=A \mathbf{v}+C(\mathbf{v}, \mathbf{v})+\bar{B} p \in \mathbf{H}^{-1}(\Omega) \\
& F^{2}(\mathbf{v}, p, \mathbf{g})=B \mathbf{v} \in L_{0}^{2}(\Omega) \\
& F^{3}(\mathbf{v}, p, \mathbf{g})=\gamma_{e} \mathbf{v}-\mathbf{v}_{\infty} \in \mathbf{H}^{1 / 2}\left(\Gamma_{e}\right) \\
& F^{4}(\mathbf{v}, p, \mathbf{g})=\gamma_{c} \mathbf{v}-\mathbf{g} \in \mathbf{H}^{1 / 2}\left(\Gamma_{c}\right)
\end{aligned}
$$

For economy, we say $\mathbf{F}: \mathbf{X} \times \mathcal{U} \rightarrow \mathbf{Y}$. Thus, our constraint (2.1)-(2.4) is

$$
\mathbf{F}=0 \in \mathbf{H}^{-1}(\Omega) \times L_{0}^{2}(\Omega) \times \mathbf{H}^{1 / 2}\left(\Gamma_{e}\right) \times \mathbf{H}^{1 / 2}\left(\Gamma_{c}\right)
$$


or

$$
\mathbf{F}=0 \in \mathbf{Y} .
$$

We say that the set $\mathcal{U}$ has property $C$ at $(\hat{\mathbf{v}}, \hat{\mathbf{g}})$ if for any nonzero solution $(\boldsymbol{\xi}, \sigma)$ of the system

$$
\begin{aligned}
-\nu \delta \xi_{i}+\xi_{j} \frac{\partial \hat{v}_{j}}{\partial x_{1}}-\hat{v}_{j} \frac{\partial \xi_{i}}{\partial x_{j}}+\frac{\partial \sigma}{\partial x_{i}} & =0 \\
\nabla \cdot \boldsymbol{\xi} & =0 \\
\left.\boldsymbol{\xi}\right|_{\Gamma} & =0
\end{aligned}
$$

we can find $\mathrm{g} \in \mathcal{U}$ such that

$$
\int_{\Gamma_{C}} \mathbf{T}(\boldsymbol{\xi}) \cdot \mathbf{n} \cdot(\mathbf{g}-\hat{\mathbf{g}}) d \Gamma<0 .
$$

Convention will have it that property $\mathrm{C}$ is to hold vacuously if there are no nonzero solutions of (2.6).

Remark. As in [3] we can show that $\mathcal{U}=\mathbf{H}^{1 / 2}(\Gamma)$ has property $\mathrm{C}$ (this is the case where the control is not constrained). This conclusion is seen to be equivalent to the following uniqueness result: If (2.7) is not true, then $\mathbf{T}(\boldsymbol{\xi})=0$ and if $\boldsymbol{\xi}$ is a solution to $(2.6)$, then $\boldsymbol{\xi}=0$.

We will use the notation $\left.D_{1} \mathbf{F}(\hat{\mathbf{v}}, \hat{\mathbf{g}})=D_{(u, p)} \mathbf{F}(\hat{\mathbf{v}}, \hat{p}, \hat{\mathbf{g}})\right)=\hat{F}, \mathbf{W}=(\mathbf{w}, q), \quad \mathbf{Z}=$ $\operatorname{image}(\hat{F}), \quad \mathbf{F}(\mathbf{v}, p, \mathcal{U})=$ image of the map $\mathbf{g} \rightarrow \mathbf{F}(\mathbf{v}, p, \mathbf{g}), \quad \mathbf{g} \in \mathcal{U}, \quad \mathbf{F}(\mathcal{U})=$ $\mathbf{F}(\hat{\mathbf{v}}, \hat{p}, \mathcal{U})$; note that $\mathbf{F}(\mathcal{U})$ is convex since $\mathbf{F}$ depends linearly on the control.

We are now in a position to state the main result of this section.

THEOREM 1. Let $(\hat{\mathbf{v}}, \hat{\mathbf{g}})$ be a local minimum (in the sense of Definition 3 ). Then there exists a nonzero Lagrange multiplier,

$$
\begin{aligned}
& \mathbb{Z}=\left(\boldsymbol{\xi}, \sigma, \boldsymbol{\zeta}_{e}, \boldsymbol{\zeta}_{c}\right) \in\left[\mathbf{H}^{-1}(\Omega) \times L_{0}^{2}(\Omega) \times \mathbf{H}^{1 / 2}\left(\Gamma_{e}\right) \times \mathbf{H}^{1 / 2}\left(\Gamma_{c}\right)\right]^{\prime} \\
&=\mathbf{H}_{0}^{1}(\Omega) \times L_{0}^{2}(\Omega) \times \mathbf{H}^{-1 / 2}\left(\Gamma_{e}\right) \times \mathbf{H}^{-1 / 2}\left(\Gamma_{c}\right) \\
&\left(\text { or }=\mathbf{H}_{0}^{1}(\Omega) \times L_{0}^{2}(\Omega) \times\left(\mathbf{H}_{0}^{1 / 2}\left(\Gamma_{(1)}\right)\right)^{\prime} \times\left(\mathbf{H}_{0}^{1 / 2}\left(\Gamma_{c}\right)\right)^{\prime}\right)
\end{aligned}
$$

(the dual spaces depend on the specified (BVP)), so that if $\mathcal{U}$ satisfies property $\mathrm{C}$ at $(\hat{\mathbf{v}}, \hat{\mathbf{g}})$, then $\hat{\mathbb{Z}}$ solves the variational system

$$
<J^{\prime}(\hat{\mathbf{v}}), \mathbf{w}>+<\hat{\mathbb{Z}}, D_{1} \mathbf{F}(\hat{\mathbf{v}}, \hat{\mathbf{g}})(\mathbf{w}, q)>=0, \quad \forall \mathbf{W} \in \mathbf{H}^{1}(\Omega) \times L_{0}^{2}(\Omega) .
$$

If property $\mathrm{C}$ is not valid then $\hat{\mathbb{Z}}$ is a nonzero solution to

$$
<\hat{\mathbb{Z}}, D_{1} \mathbf{F}(\hat{\mathbf{v}}, \hat{\mathbf{g}}) \mathbf{W}>=0, \quad \forall \mathbf{W} \in \mathbf{H}^{1}(\Omega) \times L_{0}^{2}(\Omega) ;
$$

in either case, $\hat{\mathbb{Z}}$ satisfies the variational inequality

$$
<\hat{\mathbf{Z}}, \mathbf{F}(\hat{\mathbf{v}}, \hat{p}, \mathbf{g})>\geq 0, \quad \forall \mathbf{g} \in \mathcal{U}
$$

The inequality (2.10) will henceforth be referred to as the minimum principle. The Lagrangian for this problem is

$$
\begin{aligned}
L=J(\mathbf{v})+<\mathbb{Z}, & F(\mathbf{v}, p, \mathbf{g})>=a(\mathbf{v}, \mathbf{v})+a(\mathbf{v}, \boldsymbol{\xi})+c(\mathbf{v}, \mathbf{v}, \boldsymbol{\xi}) \\
& +b(\boldsymbol{\xi}, p)+b(\mathbf{v}, \sigma)+<\boldsymbol{\zeta}_{1}, \gamma_{e} \mathbf{v}-\mathbf{v}_{\infty}>_{\Gamma}+<\boldsymbol{\zeta}_{2}, \gamma_{C} \mathbf{v}-\mathbf{g}>_{\Gamma} .
\end{aligned}
$$


The proof of Theorem 1 will rely on the following result.

THEOREM 2. (Ioffe and Tikhomirov [5]). In the notation of the foregoing, if

(a) $\mathbf{X}$ and $\mathbf{Y}$ are Banach spaces;

(b) $J \in C^{1}(\mathbf{X}), \quad D_{1} \mathbf{F}(\mathbf{v}, p) \in C^{1}(\mathbf{X})$;

(c) $\mathbf{F}(\mathbf{v}, p, \mathcal{U})$ convex;

(d) $\mathbf{Z}$ is closed in $\mathbf{Y}$ and has finite codimension;

(e) some condition of complete regularity holds (for instance, $0 \in \mathbf{Y}$ is an interior point of $\mathbf{Z}+\mathbf{F}(\mathcal{U})$ );

then there exists a $\hat{\mathbb{Z}}$ as described in Theorem 1 and satisfying (2.8) and (2.10).

Proof. For the proof see [13, Theorem P, p. 49] or [5, Theorem 3, p. 71, p. 85].

Proof of Theorem 1. We wish to apply Theorem 2 to construct a $\mathbb{Z}$ that satisfies (2.8) and (2.10). It is clear that conditions (a), (b), and (c) follow from previous observations. To show condition (d) is true, we show that $\hat{F}$ is Fredholm. If we compute the derivative,

$$
\hat{F}(\mathbf{w}, q)=D_{1} F(\hat{\mathbf{u}}, \hat{p}, \hat{\mathbf{g}})\left(\begin{array}{c}
\mathbf{w} \\
q
\end{array}\right)=\left(\begin{array}{c}
A \mathbf{w}+\bar{B} q+C(\hat{\mathbf{u}}, \mathbf{w})+C(\mathbf{w}, \hat{\mathbf{u}}) \\
B \mathbf{w} \\
\gamma_{e} \mathbf{w} \\
\gamma_{c} \mathbf{w}
\end{array}\right) .
$$

By the trace theorem, and using the ellipticity of $A$ and the inf sup property for $B$, we can see (cf. [1], [6], and [7]) that the Stokes operator

$$
\left(\begin{array}{cc}
A & \bar{B} \\
B & 0 \\
\gamma & 0
\end{array}\right)
$$

is an isomorphism from $\mathbf{H}^{1}(\Omega) \times L_{0}^{2}(\Omega) \rightarrow \mathbf{H}^{-1}(\Omega) \times L_{0}^{2}(\Omega) \times \mathbf{H}^{1 / 2}(\Gamma)$. (The last factor in this product of spaces is $\mathbf{H}_{0}^{1 / 2}(\Gamma)$ if the velocity is specified on the whole outer boundary, i.e., (BVC1).) We estimate the linear operators $C(\cdot, \hat{\mathbf{u}})$ and $C(\hat{\mathbf{u}}, \cdot)$ as follows. From the estimate

$$
|c(\mathbf{w}, \hat{\mathbf{u}}, \boldsymbol{\xi})| \leq\|\mathbf{w}\|_{1 / 2}\|\hat{\mathbf{u}}\|_{1}\|\boldsymbol{\xi}\|_{1},
$$

we have that $C(\cdot, \hat{\mathbf{u}})$ is continuous from $\mathbf{H}^{1 / 2}(\Omega)$ into $\mathbf{H}^{-1}(\Omega)$ and thus compact from $\mathbf{H}^{1}(\Omega)$ into $\mathbf{H}^{-1}(\Omega)$. Likewise, from the estimate

$$
|c(\hat{\mathbf{u}}, \mathbf{w}, \boldsymbol{\xi})| \leq\|\hat{\mathbf{u}}\|_{1}\|\mathbf{w}\|_{1}\|\boldsymbol{\xi}\|_{1 / 2}
$$

we see that $C(\hat{\mathbf{u}}, \cdot)$ is continuous from $\mathbf{H}^{1}(\Omega)$ into $\mathbf{H}^{-1 / 2}(\Omega)$ and thus compact from $\mathbf{H}^{1}(\Omega)$ into $\mathbf{H}^{-1}(\Omega)$. Thus, $\hat{F}=D_{1} \mathbf{F}(\hat{\mathbf{u}}, \hat{p}, \hat{\mathbf{g}})$ is a Fredholm operator. Since $\mathbf{Z}$ is now known to be closed and with finite codimension $M$ (being the dimension of the space of solutions of (2.6)), we can write

$$
\mathbf{Y}=\mathbf{Z} \oplus \mathbf{E}
$$

Property $\mathrm{C}$ expresses a condition of complete regularity (assumption (e) in Theorem 2); to see this, note that, under the assumption that property $\mathrm{C}$ holds, if $y \in \mathbf{Y}$, then there exist $\left(\mathbf{w}_{1}, q_{1}\right) \in \mathbf{X}, \mathbf{g} \in \mathcal{U}, \lambda \geq 0$ such that

$$
y=\hat{F}\left(\mathbf{w}_{1}, q_{1}\right)+\lambda(\hat{\mathbf{v}}, \hat{p}, \mathbf{g}) .
$$


Let $Q: \mathbf{Y} \rightarrow \mathbf{Y} / \mathbf{Z} \cong \mathbf{E}$ be the canonical mapping. If (2.11) does not hold, then taking into account the finite dimensionality of $\mathbf{E}$ and the convexity of $\mathbf{F}(\mathcal{U})$, we can find $y \in \mathbf{Y}$ such that

$$
Q y \cdot Q \mathbf{F}(\hat{\mathbf{v}}, \hat{p}, \mathbf{g}) \leq 0, \quad \forall \mathbf{g} \in \mathcal{U} .
$$

Define $\lambda=-Q y /|Q y|$ and $\Lambda=Q^{*} \lambda$, then clearly, $\Lambda \in \mathbf{Z}^{\perp}$ and $\langle\Lambda, \mathbf{F}(\mathcal{U})>\geq 0$, i.e., property $\mathrm{C}$ does not hold. Thus, we can find a finite number of $\mathbf{g}_{i} \in \mathcal{U}, 1 \leq i \leq N$, such that $\sum \mathbf{F}\left(\hat{\mathbf{v}}, \hat{p}, \mathbf{g}_{i}\right)=\hat{F} \mathbf{W}^{\prime}$ for some $\mathbf{W}^{\prime}=\left(\mathbf{w}^{\prime}, q^{\prime}\right) \in \mathbf{X}$, and so that the mapping

$$
\left(\mathbf{w}, q, \lambda_{1}, \ldots, \lambda_{n}\right) \mapsto \hat{F}(\mathbf{w}, q)+\sum \lambda_{i} \mathbf{F}\left(\hat{\mathbf{v}}, \hat{p}, \mathbf{g}_{i}\right)
$$

defined on $\mathbf{X} \times R_{+}^{N}$ is onto $\mathbf{Y}$. Let $\epsilon$ be given and let $\left(\mathbf{w}_{0}, q_{0}, \mathbf{g}_{0}\right)$ be such that

$$
\hat{F}\left(\mathbf{w}_{0}, q_{0}\right)+\mathbf{F}\left(\hat{\mathbf{v}}, \hat{p}, \mathbf{g}_{0}\right)=0 .
$$

Then the mapping

$$
\begin{aligned}
G_{\epsilon}(\mathbf{v}, p, \mathbf{a})= & \left(1-a_{0}-\epsilon \sum a_{i}\right) \mathbf{F}(\hat{\mathbf{v}}+\mathbf{v}, \hat{p}+p, \hat{\mathbf{g}}) \\
& +a_{0} \mathbf{F}\left(\hat{\mathbf{v}}+\mathbf{v}, \hat{p}+p, \mathbf{g}_{0}\right)+\epsilon \sum a_{i} \mathbf{F}\left(\hat{\mathbf{v}}+\mathbf{v}, \hat{p}+p, \mathbf{g}_{i}\right)
\end{aligned}
$$

defined on $\mathbf{X} \times R_{+}^{N+1}$, is smooth at the origin and $D G(0,0,0)$ is surjective. Ee may now continue with the proof of Theorem 2 given in [5, p. 87] or [13, p. 51] to construct a $\hat{\mathbb{Z}}$ that satisfies $(2.8)$ and $(2.10)$.

The stationarity condition $(2.8)$ gives

$$
\begin{aligned}
0=a(\hat{\mathbf{v}}, \mathbf{w})+a(\mathbf{w}, \boldsymbol{\xi})+c(\hat{\mathbf{v}}, \mathbf{w}, \boldsymbol{\xi})+c(\mathbf{w}, \hat{\mathbf{v}}, \boldsymbol{\xi})+ \\
\qquad(\boldsymbol{\xi}, q)+b(\mathbf{w}, \sigma)+<\boldsymbol{\zeta}_{1}, \gamma_{e} \mathbf{w}>+<\boldsymbol{\zeta}_{2}, \gamma_{c} \mathbf{w}>
\end{aligned}
$$

The minimum principle $(2.10)$, reduces in our case to the variational inequality

$$
<\zeta_{2}, \hat{\mathbf{g}}-\mathbf{g}>\geq 0 \quad \forall \mathbf{g} \in \mathcal{U}
$$

To get a (formal) expression for the multiplier $\boldsymbol{\zeta}_{2}$, we integrate by parts to obtain the system of equations for the adjoint variables

$$
\begin{aligned}
-\nu \Delta \hat{\xi}_{i}+\hat{\xi}_{j} \frac{\partial \hat{v}_{j}}{\partial x_{i}}-\hat{v}_{j} \frac{\partial \hat{\xi}_{i}}{\partial x_{j}}+\frac{\partial \hat{\sigma}}{\partial x_{i}} & =-\nu \Delta \hat{v}_{i} \\
\nabla \cdot \hat{\boldsymbol{\xi}} & =0 \\
\left.\hat{\boldsymbol{\xi}}\right|_{\Gamma} & =0 \\
\hat{\boldsymbol{\zeta}}_{1} & =-\left.(\mathbf{T}(\hat{\mathbf{v}})+\mathbf{T}(\hat{\boldsymbol{\xi}}))\right|_{\Gamma_{e}} \cdot \mathbf{n} \\
\hat{\boldsymbol{\zeta}}_{2} & =-\left.(\mathbf{T}(\hat{\mathbf{v}})+\mathbf{T}(\hat{\boldsymbol{\xi}}))\right|_{\Gamma_{c}} \cdot \mathbf{n} .
\end{aligned}
$$

Thus, the minimum principle reduces to

$$
\int_{\Gamma_{c}}[\mathbf{T}(\hat{\mathbf{v}})+\mathbf{T}(\hat{\boldsymbol{\xi}})] \cdot \mathbf{n} \cdot(\mathbf{g}-\hat{\mathbf{g}}) d \Gamma \geq 0, \quad \forall \mathbf{g} \in \mathcal{U}
$$


Note that this condition is equivalent to:

$$
\begin{aligned}
& \text { choose } \hat{\mathbf{g}} \in \mathcal{U} \text { that solves } \\
& \max _{\hat{\mathbf{g}} \in \mathcal{U}} \min _{\mathbf{g} \in \mathcal{U}} \int_{\Gamma}[\mathbf{T}(\mathbf{v})+\mathbf{T}(\boldsymbol{\xi})] \cdot \mathbf{n} \cdot(\mathbf{g}-\hat{\mathbf{g}}) d \Gamma
\end{aligned}
$$

In the case where property $\mathrm{C}$ does not hold it follows immediately that there exists

$$
\begin{aligned}
\mathbb{Z}=\left(\boldsymbol{\xi}, \boldsymbol{\sigma}, \boldsymbol{\zeta}_{e}, \boldsymbol{\zeta}_{c}\right) & \in\left[\mathbf{H}^{-1}(\Omega) \times L_{0}^{2}(\Omega) \times \mathbf{H}^{1 / 2}(\Gamma) \times \mathbf{H}^{1 / 2}(\Gamma)\right]^{\prime} \\
& =\mathbf{H}_{0}^{1}(\Omega) \times L_{0}^{2}(\Omega) \times \mathbf{H}^{-1 / 2}(\Gamma) \times \mathbf{H}^{-1 / 2}(\Gamma) \\
& \left(\text { or }=\mathbf{H}_{0}^{1}(\Omega) \times L_{0}^{2}(\Omega) \times\left(\mathbf{H}_{0}^{1 / 2}\left(\Gamma_{(1)}\right)\right)^{\prime} \times\left(\mathbf{H}_{0}^{1 / 2}\left(\Gamma_{c}\right)\right)^{\prime}\right)
\end{aligned}
$$

that satisfies the system of equations (2.9), i.e.,

$$
\begin{aligned}
-\nu \Delta \hat{\xi}_{i}+\hat{\xi}_{j} \frac{\partial \hat{v}_{j}}{\partial x_{i}}-\hat{v}_{j} \frac{\partial \hat{\xi}_{i}}{\partial x_{j}}+\frac{\partial \hat{\sigma}}{\partial x_{i}} & =0 \\
\nabla \cdot \hat{\boldsymbol{\xi}} & =0 \\
\left.\hat{\boldsymbol{\xi}}\right|_{\Gamma} & =0 \\
\hat{\boldsymbol{\zeta}}_{2} & =-\left.\mathbf{T}(\hat{\boldsymbol{\xi}})\right|_{\Gamma_{c}} \cdot \mathbf{n}
\end{aligned}
$$

and

$$
\int_{\Gamma_{c}} \mathbf{T}(\hat{\boldsymbol{\xi}}) \cdot \mathbf{n} \cdot(\mathbf{g}-\hat{\mathbf{g}}) d \Gamma \geq 0, \quad \forall \mathbf{g} \in \mathcal{U}
$$

thus inequality (2.10) is seen to hold in all cases.

Let us see what Theorem 1 gives us in the case where $\mathcal{U}$ is given more structure. Suppose that $\mathcal{U}$ is given by

$$
\mathcal{U}=\left\{\mathbf{g} \in \mathbf{H}^{1 / 2}: \mathbf{g} \in \mathbf{A}(\mathbf{s}), \quad \text { for almost all } \mathbf{s} \in \Gamma_{c}\right\}
$$

where $\mathbf{A}(\mathbf{s})$ is a compact, convex subset of $R^{n}$ for all $\mathbf{s}$. If we consider the sets

$$
\mathcal{G}_{s_{0}, \epsilon}=\left\{\mathbf{g} \in \mathcal{U} \ni \mathbf{g}=\hat{\mathbf{g}} \text { on } \Gamma_{s_{0}, \epsilon}=\Gamma-\Gamma \cap B\left(\mathbf{s}_{0}, \epsilon\right)\right\}
$$

then our condition reduces to

$$
\int_{\Gamma_{s_{0}, \epsilon}}[\mathbf{T}(\hat{\mathbf{v}})+\mathbf{T}(\hat{\boldsymbol{\xi}})] \cdot \mathbf{n} \cdot(\mathbf{g}-\hat{\mathbf{g}}) d \Gamma \geq 0, \quad \forall \mathbf{g} \in \mathcal{G}_{s_{0}, \epsilon}, \quad \forall \mathbf{s}_{0} \in \Gamma_{c}, \quad \forall \epsilon>0
$$

But since $\mathbf{s}_{0} \in \Gamma_{c}, \epsilon>0$ are arbitrary, we find that the pointwise condition

$$
[\mathbf{T}(\hat{\mathbf{v}})(\mathbf{s})+\mathbf{T}(\hat{\boldsymbol{\xi}})(\mathbf{s})] \cdot \mathbf{n} \cdot(\mathbf{g}-\hat{\mathbf{g}}) \geq 0, \quad \forall \mathbf{g}(\mathbf{s}) \in \mathbf{A}(\mathbf{s})
$$

holds for almost all $\mathbf{s} \in \Gamma_{c}$.

3. Finite-dimensional control. Suppose that $\mathcal{U}$ is a compact convex subset of $T$, an $m$-dimensional subspace of $\mathbf{H}^{1 / 2}(\Gamma)$ spanned by the $m$ functions $\left\{\boldsymbol{\phi}^{k}\right\}_{1}^{m}$. For the sake of unity of presentation we will assume that property $\mathrm{C}$ holds, although everything is true mutatis mutandis in any case and the final recipe is the same. Let the set of 
vectors $\left\{\mathbf{w}^{k}\right\}, \quad 1 \leq k \leq m$, be a basis for $R^{m}$, which we identify with the set $\left\{\boldsymbol{\phi}^{k}\right\}$, so that $T$ can be thought of as either $R^{m}=\operatorname{span}\left\{\mathbf{w}_{k}\right\}$ or as $\operatorname{span}\left\{\boldsymbol{\phi}^{k}\right\} \subset \mathbf{H}^{1 / 2}\left(\Gamma_{c}\right)$. Thus any control vector $\mathbf{g} \in \mathcal{U}$ has a representation of the form

$$
\mathbf{g}(\mathbf{s})=\sum g_{k} \boldsymbol{\phi}^{k} \quad \text { or } \quad \mathbf{g}=\sum g_{k} \mathbf{w}^{k}
$$

in $T$.

Let $\Pi: \mathbf{H}^{1 / 2} \rightarrow T$ be the projection. Let $\jmath: T \rightarrow \mathbf{H}^{1 / 2}$ be the canonical injection. Then we can write the components, $\{\Pi \mathbf{g}\}_{k}$, as

$$
\{\Pi \mathbf{g}\}_{k}=<<\mathbf{g}, \phi^{k}>_{T} \text {. }
$$

Here we use the $\mathbf{H}^{1 / 2}$ inner product.

Recall the minimum principle (2.13). The integral on the left in that inequality is the duality relation $\mathbf{H}^{-1 / 2} \times \mathbf{H}^{1 / 2}$, which can be written

$$
<(T(\mathbf{v})+T(\boldsymbol{\xi})) \cdot \mathbf{n}, \jmath \Pi(\mathbf{g}-\hat{\mathbf{g}})>
$$

for $\mathbf{g} \in T$. This equals

$$
<\jmath^{\prime}(\mathbf{T}+\mathbf{T}), \Pi(\mathbf{g}-\hat{\mathbf{g}})>
$$

which is the duality pairing of $T^{\prime} \times T$. Thus the minimum principle is

$$
<\jmath^{\prime}(\mathbf{T}(\hat{\mathbf{v}})+\mathbf{T}(\hat{\boldsymbol{\xi}})) \cdot \mathbf{n},(\mathbf{g}-\hat{\mathbf{g}})>\geq 0, \quad \forall \mathbf{g} \in \mathcal{U} .
$$

(This is duality pairing in $T^{\prime} \times T$.) Let us give $T^{\prime}$ the dual basis:

$$
\text { either }\left\{\boldsymbol{\omega}_{k}\right\}, \quad \text { or } \quad\left\{\boldsymbol{\psi}^{k}\right\}
$$

where

$$
<\omega_{k}, \mathbf{w}_{j}>=\delta_{k j}, \quad<\boldsymbol{\psi}^{k}, \boldsymbol{\phi}^{j}>=\delta_{k j} .
$$

The operator dual to $\jmath, \jmath^{\prime}: \mathbf{H}^{-1 / 2} \rightarrow T^{\prime}$ is a projection since $T$ is closed. Thus we can define components

$$
\eta^{k}=<<\jmath^{\prime}(\mathbf{T}(\hat{\mathbf{v}})+\mathbf{T}(\hat{\boldsymbol{\xi}})), \boldsymbol{\psi}^{k}>_{-1 / 2}
$$

so that the minimum principle (2.13) now becomes

$$
\sum_{k} \eta^{k}(g-\hat{g})_{k} \geq 0, \quad \forall \mathbf{g}=\left\{g_{k}\right\} \in \mathcal{U}
$$

Let us look at the special case where $\mathcal{U}$ is a parallelpiped

$$
\left\{a_{k} \leq g_{k} \leq b_{k}\right\}
$$

then the minimum principle is seen to resolve itself as $m$ independent inequations

$$
\eta^{k}\left(h-\hat{g}_{k}\right) \geq 0, \quad \forall h \in\left[a_{k}, b_{k}\right]
$$

and thus we have the following prescription for the optimal control:

$$
\begin{aligned}
& \text { if } \eta^{k}>0 \text { then } \hat{g}_{k}=a_{k} \\
& \text { if } \eta^{k}<0 \text { then } \hat{g}_{k}=b_{k} .
\end{aligned}
$$


If $\eta^{k}=0$, then the Lagrange necessary condition gives no information about $\hat{g}_{k}$.

We see that in this procedure, the $\eta_{k}$ would seem to have to be computed in the $\mathbf{H}^{-1 / 2}$ inner product, which may pose computational difficulties. It turns out that by choosing the $\left\{\phi^{k}\right\}$ properly we can avoid this situation. In particular, suppose that the $\left\{\boldsymbol{\phi}^{k}\right\}$ form an orthonormal set in $\mathbf{H}^{1 / 2}$. Let $\Lambda: \mathbf{H}^{1 / 2} \rightarrow \mathbf{H}^{-1 / 2}$ be the canonical isomorphism; then

$$
<\Lambda \phi^{k}, \phi^{j}>=\delta_{k j}
$$

where this is duality. This means that we can choose the sequence $\left\{\boldsymbol{\psi}^{k}\right\}=\left\{\Lambda \boldsymbol{\phi}^{k}\right\}$, and

$$
\begin{aligned}
\eta_{k}=<<\mathbf{T}(\mathbf{v})+\mathbf{T}(\boldsymbol{\xi}), \boldsymbol{\psi}^{k} \cdot>_{-1 / 2} & =<\mathbf{T}(\hat{\mathbf{v}})+\mathbf{T}(\boldsymbol{\xi}), \Lambda \boldsymbol{\phi}^{k}>_{-1 / 2} \\
& =<\mathbf{T}(\hat{\mathbf{v}})+\mathbf{T}(\boldsymbol{\xi}), \boldsymbol{\phi}^{k}>
\end{aligned}
$$

in other words,

$$
\eta^{k}=\int_{\Gamma_{c}}[\mathbf{T}(\hat{\mathbf{v}})+\mathbf{T}(\hat{\boldsymbol{\xi}})] \cdot \mathbf{n} \cdot \boldsymbol{\phi}^{k} d \Gamma .
$$

In the degenerate case, the prescription (3.1) continues to hold, although in that case, we have

$$
\eta^{k}=\int_{\Gamma_{c}} \mathbf{T}(\hat{\boldsymbol{\xi}}) \cdot \mathbf{n} \cdot \boldsymbol{\phi}^{k} d \Gamma
$$

4. Application to drag reduction via discrete blow holes. Let us consider the situation where there are $m$ subregions of $\Gamma_{c}$, say the set $\left\{\Gamma_{k}\right\}$, such that

$$
\Gamma_{c}-\cup \Gamma_{k} \neq \emptyset, \quad \overline{\Gamma_{k} \cup \Gamma_{j}}=\emptyset \quad k \neq j
$$

Consider a set of vector-valued functions, $\left\{\boldsymbol{\phi}^{k}\right\}$ in $\mathbf{H}^{1 / 2}\left(\Gamma_{c}\right)$ such that

$$
\operatorname{supp} \phi^{k} \subset \Gamma_{k}, \quad \forall k
$$

so, clearly, these functions are orthogonal; we also want them normalized in the $\mathbf{H}^{1 / 2}$ norm.

We will define our admissibility set for the control as

$$
\mathcal{U}=\left\{\mathbf{g} \in \mathbf{H}^{1 / 2}\left(\Gamma_{c}\right): \mathbf{g}=\sum \gamma_{k} \boldsymbol{\phi}^{k}, a_{k} \leq \gamma_{k} \leq b_{k}, a_{k}, b_{k} \in R, \quad \forall k\right\}
$$

Now we can apply the framework of the last section. Let $T$ be identified with Euclidean $m$-space, with the usual basis; i.e.,

$$
\left\{\mathbf{e}_{1}, \cdots, \mathbf{e}_{m}\right\}
$$

The minimum principle is

$$
\sum_{k} \int_{\Gamma_{k}}[\mathbf{T}(\hat{\mathbf{v}})+\mathbf{T}(\boldsymbol{\xi})] \cdot \mathbf{n} \cdot(\mathbf{g}-\hat{\mathbf{g}}) d \Gamma \geq 0, \quad \forall \mathbf{g} \in \mathcal{U}
$$

which reduces to

$$
\sum_{k} \eta^{k}(g-\hat{g})_{k} \geq 0, \quad \forall \mathbf{g}=\left\{g_{k}\right\} \in \mathcal{U}
$$


where

$$
\eta^{k}=\int_{\Gamma_{k}}[\mathbf{T}(\hat{\mathbf{v}})+\mathbf{T}(\boldsymbol{\xi})] \cdot \mathbf{n} \cdot \boldsymbol{\phi}^{k} d \Gamma .
$$

Note that an apparent difficulty in implementation is the normalization of the basis vectors in the $\frac{1}{2}$ norm. Suppose that, instead,

$$
\int_{\Gamma}\left[\left(\phi_{1}^{k}\right)^{2}+\ldots+\left(\phi_{n}^{k}\right)^{2}\right] d \Gamma=1, \quad \forall k
$$

so that they are actually an orthonormal set in $\mathbf{L}^{2}(\Gamma)$. But this is also the duality pairing, thus we can choose the $\boldsymbol{\psi}$ 's to be the $\phi$ 's. But in this case,

$$
\eta_{k}=<<\mathbf{T} ; \boldsymbol{\phi}^{k}>_{-1 / 2}=<\mathbf{T}, \Lambda^{-1} \boldsymbol{\phi}^{k}>
$$

which means that the $\eta$ 's would be difficult to compute. In any case, the question of computing the $\eta^{k}$ is moot, unless we know whetheror not property $\mathrm{C}$ is to hold. The question of efficiently computing the optimum is far from satisfactorily settled. The formula (3.1) gives us the structure of the optimal solution, but it may be prohibitively expensive to have to visit each vertex of the $m$-cube. Note that the $\frac{1}{2}$ norm of the basis functions is not necessarily difficult to calculate. A reasonable choice of basis functions, at least for $a_{k}$ and $b_{k}$ small, are the piecewise linear functions, i.e., hat functions over the holes. These $\mathbf{H}^{1 / 2}$-norms can be computed. For example, if $n=2$, and $\Gamma_{1}=[0, b]$, and

$$
\phi_{1}^{1}= \begin{cases}(2 h / b) x & \text { if } x \leq b / 2 \\ 2 h(1-x / b) & \text { if } x>b / 2\end{cases}
$$

then,

$$
\left\|\phi_{1}^{1}\right\|_{1 / 2}^{2}=h^{2}\left[b / 6+16 / b^{2}+8(\ln (2)-1 / 4)\right] .
$$

Remark 1. Note that the vector $\left(\eta^{1}, \ldots, \eta^{m}\right)$ is matrix representation for a Jacobian of $J^{\prime}(\hat{\mathbf{g}})$. Thus, if $\eta^{j}=0$ then $\hat{g}^{j} \in\left[a_{k}, b_{k}\right]$ is arbitrary, and we have a one-parameter family of extremals, parametrized by $\left[a_{j}, b_{j}\right]$. In the application at hand, this means that the $j$ th control hole could be removed without effect on the functional. It would be interesting to know whether this kind of degeneracy affects the convergence of the approximation.

Remark 2. Care must be taken to make the problem well-posed, not only in the sense of existence and regularity, but also with respect to the control. Degeneracies can arise in several ways: if property $\mathrm{C}$ does not hold then it is seen that the necessary conditions do not involve the cost functional; this means that the constraint set does not have the structure that we want; it is not a manifold, or solutions of the constraint equations are so sparse that a variational approach is not useful. On the other hand, if $\mathbf{g} \in \mathcal{U}$ supplies an unconstrained minimum (for example, if $\mathbf{v}_{\infty}=0$ and $\mathbf{g}=0$, then $J^{\prime}(\hat{\mathbf{v}}) \equiv 0$, and the constraint is superfluous; in which case a result such as (3.1) would be useless. Note that we do not have to explicitly rule out this case, for then, as can be deduced from the previous remark, $\eta_{k}=0$, for all $k$.

5. A simple computational example. Recall that the problem to be discretized is given by

$$
\begin{aligned}
& a(\mathbf{v}, \mathbf{w})+c(\mathbf{v}, \mathbf{v}, \mathbf{w})+b(\mathbf{w}, p)=0, \quad \forall \mathbf{w} \in \mathbf{H}_{0}^{1}(\Omega) \\
& b(\mathbf{v}, q)=0, \quad \forall q \in L_{0}^{2}(\Omega) \\
& \left.\mathbf{v}\right|_{\Gamma_{k}}=\mathbf{g}_{k} \\
& \left.\mathbf{v}\right|_{\Gamma-\left(\cup \Gamma_{k}\right) \cup \Gamma_{(1)}}=\mathbf{w}_{0} \\
& \left.\mathbf{T}(\mathbf{v}) \cdot \mathbf{n}\right|_{\Gamma_{(2)}}=0 \text {, }
\end{aligned}
$$


where $\mathbf{w}_{0}$ is a prescribed function. For the simple example given below we did not use the adjoint system in the updating procedure, only the theoretical fact that the optimal solution is bang-bang i.e., given by (3.1) and (3.2). The discretization is effected using a standard finite element algorithm (cf. [7]). Thus, we choose finite element subspaces

$$
\mathbf{V}^{h} \subset \mathbf{H}^{1}(\Omega)
$$

and

$$
S^{h} \subset L_{0}^{2}(\Omega)
$$

and require that $\mathbf{v}^{h} \in \mathbf{V}^{h}$ and $p^{h} \in S^{h}$ satisfy

$$
\begin{aligned}
& a\left(\mathbf{v}^{h}, \mathbf{w}^{h}\right)+c\left(\mathbf{v}^{h}, \mathbf{v}^{h}, \mathbf{w}^{h}\right)+b\left(\mathbf{w}^{h}, p^{h}\right)=0, \quad \forall \mathbf{w}^{h} \in \mathbf{V}^{h} \\
& b\left(\mathbf{v}^{h}, q^{h}\right)=0, \quad \forall q^{h} \in S^{h} \\
& \left.\mathbf{v}^{h}\right|_{\Gamma_{k}}=\mathbf{g}_{k}^{h} \\
& \left.\mathbf{v}^{h}\right|_{\Gamma-\left(\cup \Gamma_{k}\right) \cup \Gamma_{(1)}}=\mathbf{w}_{0}^{h} \text {, }
\end{aligned}
$$

where $\mathbf{g}_{k}^{h}$ and $\mathbf{w}_{0}^{h}$ are suitable approximations to $\mathbf{g}_{k}$ and $\mathbf{w}_{0}$, respectively. (See the discussion above.)

The particular computational problem we consider here is described in Fig. 1.

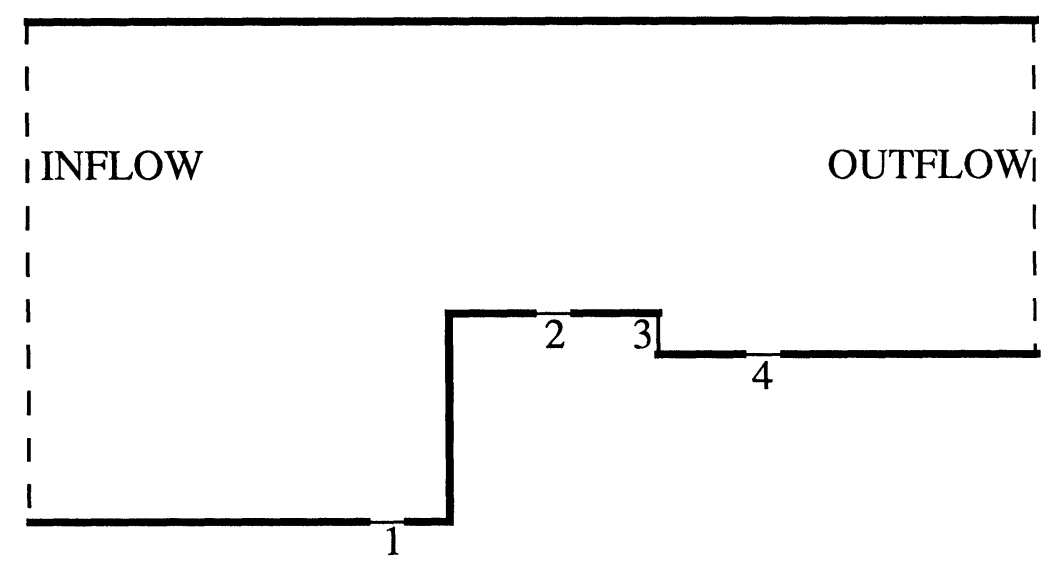

Fig. 1. Geometry and hole placemnt for flow over a forward facing step.

Here, $\Gamma_{k}, k=1,2,3,4$, are the regions indicated and $\mathbf{w}_{0}$ is zero on the top and bottom portions of $\Gamma-\cup_{k} \Gamma_{k}$ and nonzero on the inflow and outflow portions. This problem is of use in viscous drag reduction studies. The step represents a protuberance on a wing, e.g., due to struts, rivets, etc. These obstacles can trip separation or accelerate transition to turbulence. Our studies examine how the injection or suction of fluid can ameliorate the negative influence of the step. We use the Taylor-Hood element pair as amended by [12]. Continuous piecewise quadratic polynomials with respect to a triangulation of the flow domain are employed for the velocity components. For the pressure approximation, we use continuous piecewise linear polynomials with respect 
to the same triangulation, augmented by a piecewise constant in each triangle. This locally mass conserving element pair is known to provide the error estimate (see [12])

$$
\left\|\mathbf{v}-\mathbf{v}^{h}\right\|_{0}+h\left(\left\|\mathbf{v}-\mathbf{v}^{h}\right\|_{1}+\left\|p-p^{h}\right\|_{0}\right) \leq C h^{s+1}\left(\|\mathbf{v}\|_{s+1}+\|p\|_{s}\right),
$$

whenever

$$
\mathbf{v} \in \mathbf{H}^{s+1}(\Omega), \quad p \in H^{s}(\Omega), \quad 1 \leq s \leq 2,
$$

where $h$ is a measure of the size of the finite element grid, and whenever $\mathbf{g}_{k}^{h}$ and $\mathbf{w}_{0}^{h}$ are accurate enough approximations. For example, the above estimate holds if we merely take $\mathbf{g}_{k}^{h}$ and $\mathbf{w}_{0}^{h}$ to be the interpolant, in $\mathbf{V}^{h}$ restricted to the appropriate segment of $\Gamma$, of $\mathbf{g}_{k}$ and $\mathbf{w}_{0}$ respectively.

For the computation we choose the velocity profile at the hole to satisfy

$$
\mathbf{g}_{k} \cdot \mathbf{n}=\alpha_{k} p_{2 k}, \quad \alpha_{k} \in\left[a_{k}, b_{k}\right] \quad \text { and } \quad \mathbf{g} \times \mathbf{n}=0,
$$

where $p_{2 k}$ is a quadratic polynomial vanishing at the edge of the hole. Then, our control set is determined by the appropriate parameters. From the theory presented above, we know that, for the optimal solution, $\alpha_{k}$ must be either $a_{k}$ or $b_{k}$. Thus, to determine the optimal solution, we need to solve our finite element problem with $\mathbf{g}_{k}$ given, following the above prescription. One may then compute $J(\mathbf{v})$ for each of these cases (16 in this example), and thus determine the minimum.

We choose the $a_{k}, b_{k}$ so that the maximum mass flow in or out of any hole is $1 / 12$ of the mass flow at the inflow. We compute the dissipation function with each hole blowing, sucking, or turned off. If only blowing is allowed, i.e., $a_{k}=0$, it is found that the dissipation function is minimized if only the second hole is active. If only suction is allowed, i.e., $b_{k}=0$, it is found that allowing only the third hole to be active is best. If both blowing and suction are allowed, i.e., $a_{k}=-b_{k}$, then having suction through the third hole and injection through the second hole is best.

\section{REFERENCES}

[1] L. Cattabriga, Su un problema al contorno relativo al sistema di equazione di Stokes, Rend. Mat. Sem. Univ. Padova, 31 (1961), pp. 308-340.

[2] G. Duvaut and J. Lions, Inequalities in Mechanics and Physics, Springer, Berlin, 1976.

[3] M. Gunzburger, L. Hou, And T. Svobodny, Analysis and finite element approximation of optimal control problems for the stationary Navier-Stokes equations with Dirichlet controls, to appear in Math. Model. Numer. Anal.

[4] G. Hough, Viscous Flow Drag Reduction, American Institute of Aeronautics and Astronautics, New York, 1980.

[5] A. Ioffe and V. Tikhomorov, Extremal Problems, North-Holland, Amsterdam, 1979.

[6] V. Girault And P.-A. Raviart, Finite Element Approximation of the Navier-Stokes Equations, Springer, Berlin, 1979.

[7] V. Girault and P.-A. Raviart, Finite Element Methods for Navier-Stokes Equations: Theory and Algorithms, Springer, New York, 1986.

[8] O. Ladyzhenskaya, The Mathematical Theory of Viscous Incompressible Flow, Gordon and Breach, New York, 1963.

[9] J. SERrin, Mathematical principles of classical fluid mechanics, Handbuch der Physik, VIII/1 (1959), pp. 1-125.

[10] R. Temam, Navier-Stokes Equations, North-Holland, New York, 1979.

[11] R. Tемам, Navier-Stokes Equations and Nonlinear Functional Analysis, SIAM, Philadelphia, 1983.

[12] R. ThATCher, Locally mass-conserving Taylor-Hood elements for two- and three-dimensional flow, Inter. J. Numer. Meth. Fluids, 11 (1990), pp. 341-353.

[13] V. Tikнomorov, Fundamental Principles of the Theory of Extremal Problems, Wiley, Chichester, 1982. 